\title{
Mortality Following Hip Fractures in the Elderly: Relation with Timing of Fixation, Length of Hospital Stay and Comorbidities
}

\author{
Khairul Mohd Khalid ${ }^{1}$, Nik Mohd Fatmy Nik Mohd Najmi ${ }^{1}$, Mohamad Fauzlie Yusof ${ }^{2}$ \\ ${ }^{1}$ Department of Orthopaedics, Traumatology and Rehabilitation, Kulliyyah of \\ Medicine, International Islamic University Malaysia \\ ${ }^{2}$ Department of Orthopaedics, Hospital Melaka
}

Presenter: Khairul Mohd Khalid

Introduction: The incidence of hip fractures in Malaysia was 218 per 100,000 for females and 88 per 100,000 for men and they are associated with mortality rate up to $33 \%$ at 1 year as shown by international studies. There are no local studies available in regards to mortality rate and associated factors. We aimed to determine mortality rate in elderly patient following hip fractures within 1 year. In addition, we evaluated the assocation of timing of fixation, length of hospital stay and number of comorbidities with mortality in these patients. Materials And Methods: This was a cross sectional study conducted in Hospital Melaka. All patients aged 65 and older who were admitted and underwent surgery for hip fractures from January 2014 to January 2017 were included. Patients or relatives were contacted via phone calls to determine their status of living. From the records, timing of fixation, length of hospital stay and number of comorbidities were recorded. Results: Mortality rate at 1 year was $10.55 \%$. From Simple Logistic Regression analysis, all three factors of timing of fixation, length of hospital stay and number of comorbidities were found to have significant value less than 0.25. From Multiple Logistic Regression, only length of stay was found to be significant $(p<0.0001)$. The significant variable can be interpreted as follows: a person with one day longer in hospital stay has 1.89 times the chance to be deceased at 1 year. Conclusion: This study shows $10.55 \%$ mortality rate at 1 year following hip fractures in the elderly. There were an association between mortality and timing of fixation, length of hospital stay and patients' comorbidities. 